\title{
Bifurcation Analysis of Current Mode Control Ćuk DC-DC Converter
}

\author{
Mohamed B. Debbat \\ Faculty of Sciences and Technology, \\ University of Mascara, \\ Mascara, Algeria.
}

\author{
Abdelali El Aroudi \\ Departement of Electronics, \\ Electrical and Automatic Engineering, \\ University of Rovira i Virgili, \\ Tarragona, Spain.
}

\author{
Rochdi Bouyadjra \\ Faculty of Sciences and Technology, \\ University of Mascara, \\ Mascara, Algeria.
}

\begin{abstract}
In this paper, bifurcation analysis of current mode control Ćuk DCDC converter operating in continuous conduction mode is carried out. Nonlinear discrete maps as much for 1-periodic orbit as for 2periodic orbit have been built. The stability analysis of both orbits is concerned using the Jacobian matrix and its eigenvalues. When the reference current is taken as a bifurcation parameter, it has been shown that the 1-periodic orbit loses its stability via flip bifurcation and the resulting is a stable 2-periodic orbit. By increasing the reference current further more, the 2-periodic orbit collides with a borderline and bifurcates to chaos via border collision bifurcation. A closed form expression of the borderline has been calculated.
\end{abstract}

\section{Keywords:}

DC-DC Converter Current Mode Control, Nonlinear DiscreteTime Map, Stability, Analysis Bifurcation ifx

\section{INTRODUCTION}

DC-DC converters exhibit nonlinear dynamical behavior due to switching components and action of the control feedback. They are able to present nonlinear phenomena like bifurcations and chaos[1]. Different nonlinear phenomena were discovered in different DC-DC converters under different control strategies [2] 3, 4].

The Cuk DC-DC converter is one of converters most used in many industrial applications but few works have been dedicated to study the nonlinear phenomena in this converter. In [5], this authors have demonstrated, via an experimental study, that the converter loses stability via a period-doubling bifurcation and exhibits chaos when the reference current is varied. Hopf bifurcation has been also demonstrated as an other route to chaos in this converter [6, 7, 8].

In the present paper, bifurcation analysis of current mode control (CMC) Ćuk DC-DC converter operating in continuous conduction mode $(\mathrm{CCM})$ is carried out by using a nonlinear discrete map to modeling the system, computing its Jacobian matrix and taking the reference current $I_{r e f}$ as a bifurcation parameter. The remainder of this work is organized as follows: in Section 2, the operating principle will be explained and the state equations describing the dynamic of the system will be derived. In section 3 , nonlinear discrete maps of as much for 1-periodic orbit as for 2periodic orbit will be built. Its fixed points and Jacobian matrix will be computed in order to study the stability of these orbits. Bifurcation analysis of the converter under CMC will be carried out in section 4. Finally, the conclusions of this work will be cited in Section 5.

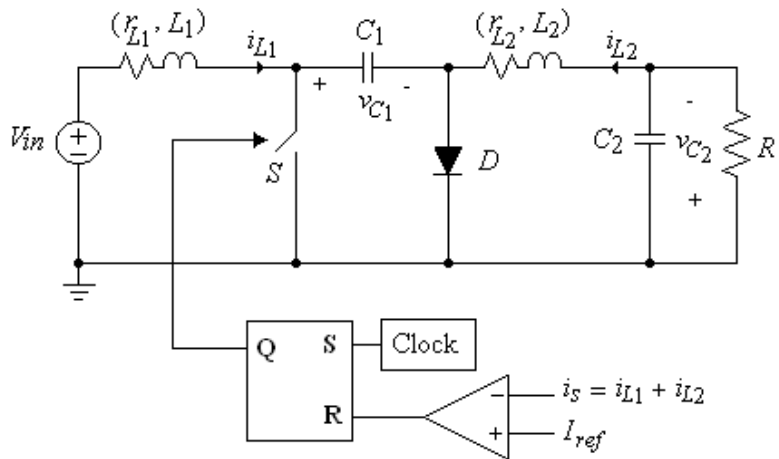

(a)

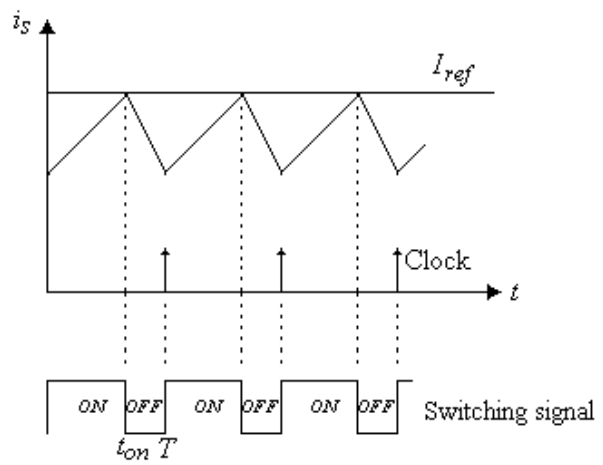

(b)

Fig. 1. (a) Bloc diagram of the current mode control Ćuk DC-DC converter. (b) A stable 1-periodic behavior.

\section{OPERATING PRINCIPAL AND STATE EQUATIONS}

The CMC Cuk DC-DC converter is shown in Fig. 1-a. It is assumed that the converter operates in CCM, both switch and diode to be ideal and ESR of inductors are included in the circuit. The converter is controlled by comparing the sum $i_{s}$ of the inductor currents $\left(i_{s}=i_{L 1}+i_{L 2}\right)$ with the reference current $I_{r e f}$. At the beginning of the cycle, the switch $S$ is turned ON. The controlled current $i_{s}$ increases until reaches the value of $I_{\text {ref }}$, the switch $S$ is turned OFF, and remains OFF until the next cycle begins (Fig. $1-b)$.

Depending on state of the switch $S$ and during one switching cycle, the Ćuk DC-DC converter has two linear configurations (phases) (Fig. 2-a and Fig. 2-b). The converter switches from configuration 1 to configuration 2 whenever the following 

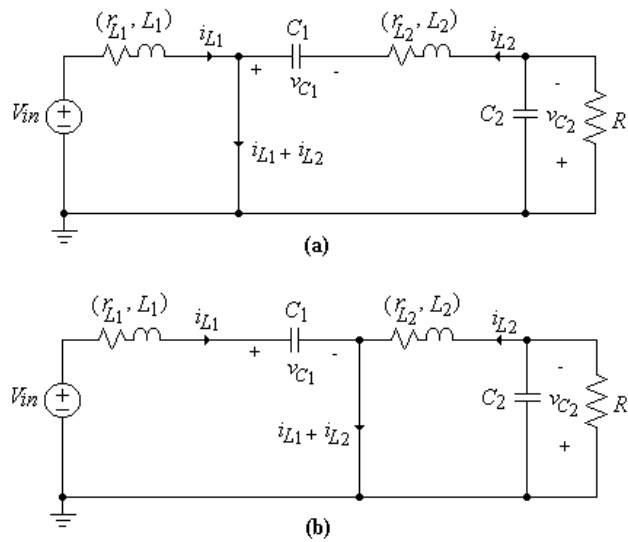

Fig. 2. (a) Topology ON: $S$ ON and $D$ OFF; (b) Topology OFF: $S$ OFF and $D O N$.

switching function

$$
\sigma(x, t):=I_{r e f}-K x
$$

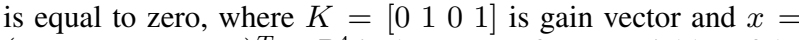
$\left(v_{C 2}, i_{L 2}, v_{C 1}, i_{L 1}\right)^{T} \in R^{4}$ is the vector of state variables of the system.

The dynamic behavior of the system, in each phase, is given by a linear differential equation, which can be described as follows:

$$
\begin{array}{ll}
\dot{x}=A_{1} x+B_{1} & \text { phase ON } \\
\dot{x}=A_{2} x+B_{2} & \text { phase OFF }
\end{array}
$$

The A's $\in R^{4 \times 4}$ and B's $\in R^{4 \times 1}$ are constants matrices during each switching intervals which are given by:

$$
\begin{gathered}
A_{1}=\left[\begin{array}{rrrr}
\frac{-1}{R C_{2}} & \frac{1}{C_{2}} & 0 & 0 \\
\frac{-1}{L_{2}} & \frac{-r_{L 2}}{L_{2}} & \frac{1}{L_{2}} & 0 \\
0 & \frac{-1}{C_{1}} & 0 & 0 \\
0 & 0 & 0 & \frac{-r_{L 1}}{L_{1}}
\end{array}\right], B_{1}=\left[\begin{array}{r}
0 \\
0 \\
0 \\
\frac{V_{i n}}{L_{1}}
\end{array}\right] \\
A_{2}=\left[\begin{array}{rrrr}
\frac{-1}{R C_{2}} & \frac{1}{C_{2}} & 0 & 0 \\
\frac{-1}{L_{2}} & \frac{-r_{L 2}}{L_{2}} & 0 & 0 \\
0 & 0 & 0 & \frac{1}{C_{1}} \\
0 & 0 & \frac{-1}{L_{1}} & \frac{-r_{L 1}}{L_{1}}
\end{array}\right], B_{2}=\left[\begin{array}{r}
0 \\
0 \\
0 \\
\frac{V_{i n}}{L_{1}}
\end{array}\right]
\end{gathered}
$$

where $V_{i n}, L_{1}, r_{L 1}, L_{2}, r_{L 2}, C_{1}, C_{2}$, and $R$ are the circuit parameters which are chosen to ensure that the converters operate in CCM.

\section{DERIVATION OF NONLINEAR DISCRETE MAP}

Because the Ćuk DC-DC converter operates cyclically, working with models that involve quantities sampled once per cycle is especially natural. The nonlinear discrete map is a suitable tool to represent the periodic behavior of the converter [9, 10, 11]. We will drive this map as much for 1-periodic orbit as for 2-periodic orbit.

\subsection{For 1-Periodic Orbit}

Let $x$ be the vector of state variables as defined previously, and let $t_{n}$ be the switching instant corresponding to when the system changes its phase from 1 to 2 . During each phase the system equations are linear and time invariant. In this case, the solution during each phase interval is available and takes the following form:

$$
x(t)=e^{A\left(t-t_{n}\right)} x\left(t_{n}\right)+\int_{t_{n}}^{t} e^{A(t-\alpha)} B d \alpha
$$

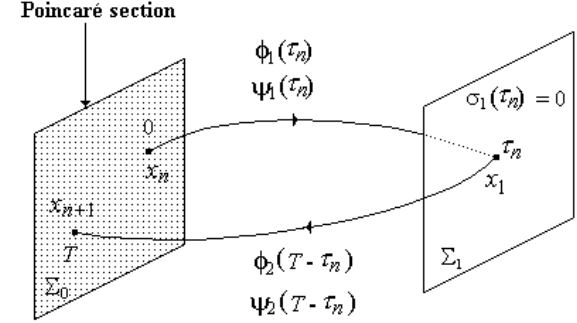

Fig. 3. Schematic mapping of 1-periodic orbit's nonlinear discrete map.

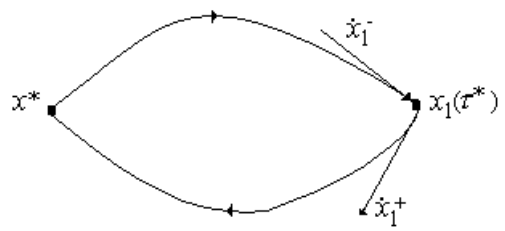

Fig. 4. Typical 1-periodic solution.

where $x\left(t_{n}\right)$ is the state vector at $t_{n}$. In order to drive the nonlinear discrete map, let us write Eq. (3) in the following form for convenience.

$$
x(t)=\Phi\left(t-t_{n}\right) x\left(t_{n}\right)+\Psi\left(t-t_{n}\right)
$$

where $\Phi(t)=e^{A_{i} t}$ and $\Psi(t)=A_{i}^{-1}(\Phi(t)-I) B_{i}$ and $I$ is the identity matrix. We will built a nonlinear discrete map (Fig. 3) in the form of a stroboscopic map which relates the state variable $x_{n}$ at instant $n T$ to $x_{n+1}$ those at instant $(n+1) T$. Using the solution of the state equation of each linear phase and linking them at the switching instant $\tau_{n}=t_{n}$, the map is expressed as follows:

$$
\begin{aligned}
x_{n+1} & :=P\left(x_{n}, \tau_{n}, p\right) \\
& =\Phi_{2}\left(T-\tau_{n}\right)\left(\Phi_{1}\left(\tau_{n}\right) x_{n}+\Psi_{1}\left(\tau_{n}\right)\right)+\Psi_{2}\left(T-\tau_{n}\right) \\
\sigma\left(x_{n}, \tau_{n}, p\right) & :=I_{\text {ref }}-K\left(\Phi_{1}\left(\tau_{n}\right) x_{n}+\Psi_{1}\left(\tau_{n}\right)\right)=0
\end{aligned}
$$

where $T$ is the switching period and $p$ is the vector of parameters.

3.1.1 Fixed points. Enforcing periodicity $\left(x_{n+1}=x_{n}=x^{*}\right)$ in the map (Eq. (5)), we obtain:

$$
\begin{gathered}
x^{*}=\left(I-\Phi_{2}\left(T-\tau^{*}\right) \Phi_{1}\left(\tau^{*}\right)\right)^{-1}\left(\Phi_{2}\left(T-\tau^{*}\right) \Psi_{1}\left(\tau^{*}\right)\right. \\
\left.+\Psi_{2}\left(T-\tau^{*}\right)\right)
\end{gathered}
$$

where $\tau^{*}$ is the switching instant corresponding to $x^{*}$ which is given by the switching equation:

$$
\sigma\left(x^{*}, \tau^{*}\right):=I_{r e f}-K\left(\Phi_{1}\left(\tau^{*}\right) x^{*}+\Psi_{1}\left(\tau^{*}\right)\right)=0
$$

Replacing $x^{*}$ in Eq. ( 7) by its expression of Eq. ( 6) we obtain the following 1-dimensional equation:

$$
\begin{aligned}
\sigma\left(\tau^{*}\right):= & I_{r e f}-K\left(\Phi _ { 1 } ( \tau ^ { * } ) \left(I-\Phi_{2}\left(T-\tau^{*}\right) \Phi_{1}\left(\tau^{*}\right)^{-1}\right.\right. \\
& \left(\Phi_{2}\left(T-\tau^{*}\right) \Psi_{1}\left(\tau^{*}\right)+\Psi_{2}\left(T-\tau^{*}\right)\right) \\
& \left.+\Psi_{1}\left(\tau^{*}\right)\right)=0
\end{aligned}
$$

Solving this equation for $\tau^{*}$ and using Eq. (6), we can obtain the fixed points $x^{*}$.

3.1.2 Jacobian matrix. Near the fixed point, the local dynamics are governed by:

$$
\tilde{x}_{n+1} \approx D P \tilde{x}_{n}
$$




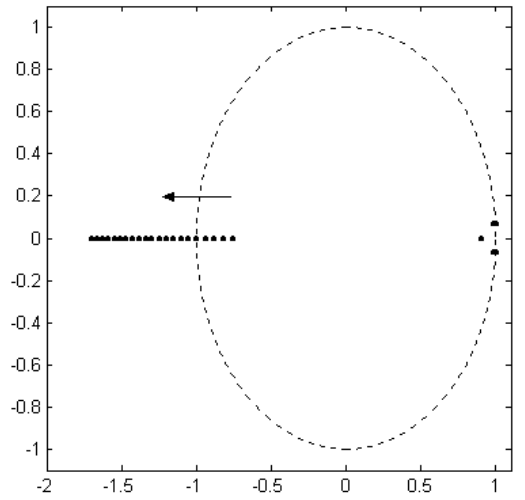

Fig. 5. The evolution of eigenvalues of the Jacobian matrix evaluated at the 1-periodic fixed points when $I_{r e f}$ is varied in the range $(2,6) \mathbf{A}$.

where $\tilde{x}_{n}=x_{n}-x^{*}$ is a small perturbation near $x^{*}$ and $D P$ is the Jacobian matrix of the map (Eq. (5)) which can be written as follow:

$$
D P=\frac{\partial P}{\partial x_{n}}-\left.\frac{\partial P}{\partial \tau_{n}}\left(\frac{\partial \sigma}{\partial \tau_{n}}\right)^{-1} \frac{\partial \sigma}{\partial x_{n}}\right|_{x^{*}, \tau^{*}}
$$

Evaluating each partial derivative and replacing it in Eq. (10), the Jacobian matrix $D P$ evaluated at $x^{*}$ becomes:

$$
D P=\Phi_{2}\left(T-\tau^{*}\right)\left(I-\frac{\left(\dot{x}_{1}^{-}-\dot{x}_{1}^{+}\right) K}{K \dot{x}_{1}^{-}}\right) \Phi_{1}\left(\tau^{*}\right)
$$

where $\dot{x}_{1}^{-}, \dot{x}_{1}^{+}$are the time derivatives of $x$ at time $\tau^{*}$ ( Fig. 4) which can be written as follow:

$$
\begin{aligned}
& \dot{x}_{1}^{-}=A_{1}\left(\Phi_{1}\left(\tau^{*}\right) x^{*}+\Psi_{1}\left(\tau^{*}\right)\right)+B_{1} \\
& \dot{x}_{1}^{+}=A_{2}\left(\Phi_{1}\left(\tau^{*}\right) x^{*}+\Psi_{1}\left(\tau^{*}\right)\right)+B_{2}
\end{aligned}
$$

3.1.3 Stability Study of 1-Periodic Orbit. Using the Jacobian matrix (Eq. (11)) developed above, we can study the stability of 1-periodic orbit when $I_{r e f}$ is taken as bifurcation parameter. $I_{r e f}$ is varied in the range $(2,6)$ A while the other circuit parameter values are fixed as follows: $V_{i n}=15 \mathrm{~V}, L_{1}=L_{2}=75$ $\mathrm{mH}, r_{L 1}=r_{L 2}=0.02 \Omega, C_{1}=C_{2}=47 \mu \mathrm{F}, R=10 \Omega$ and $T=50 \mu$ s. In Fig. 5, we show the evolution of the eigenvalues of the Jacobian matrix $D P$ evaluated at the 1-periodic fixed point when $I_{r e f}$ is taken as bifurcation parameter. We can see that as $I_{\text {ref }}$ is increased, one of the eigenvalues crosses the unit circle through $(-1,0)$ at $I_{r e f} \simeq 4 \mathrm{~A}$. This corresponds to a flip bifurcation (FB). The 1-periodic orbit loses its stability and becomes a stable 2-periodic orbit. The corresponding eigenvalues at this bifurcation are $(-1.0000 ; 0.9139 ; 0.9761 \pm 0.075 i)$.

\subsection{For 2-periodic orbit}

During the switching cycle of duration $2 T$, Cuk DC-DC converter has four phases defined by the system matrices $\left(A_{1}, B_{1}\right)$, $\left(A_{2}, B_{2}\right),\left(A_{1}, B_{1}\right)$, and $\left(A_{2}, B_{2}\right)$ respectively (Fig. 6-a). Therefore, there are three switching instants that compose the vector of switching instants $\tau_{n}=\left[t_{1, n}, t_{2, n}, t_{3, n}\right]^{T}$. The instant $t_{2, n}=T$ is imposed by the latch, while the instants $t_{1, n}, t_{3, n}$ are calculed by two switching equations $\sigma_{1}$ and $\sigma_{2}$ respectively. After defining the number of configurations and the switching instants, to derive the map, a similar procedure as in the case of 1-periodic orbit is used (Fig. 6-b).

$$
\begin{aligned}
x_{n+1}: & =P\left(x_{n}, \tau_{n}, p\right) \\
& =\Phi_{2}\left(2 T-t_{3, n}\right)\left(\Phi _ { 1 } ( t _ { 3 , n } - T ) \left(\Phi_{2}\left(T-t_{1, n}\right)\right.\right. \\
& \left(\Phi_{1}\left(t_{1, n}\right) x_{n}+\Psi_{1}\left(t_{1, n}\right)\right)+\Psi_{2}\left(T-t_{1, n}\right) \\
& \left.\quad+\Psi_{1}\left(t_{3, n}-T\right)\right)+\Phi_{2}\left(2 T-t_{3, n}\right)
\end{aligned}
$$

$$
\sigma\left(x_{n}, \tau_{n}, p\right):=\left[\begin{array}{l}
\sigma_{1}\left(x_{n}, t_{1, n}, p\right) \\
\sigma_{2}\left(x_{n}, t_{1, n}, t_{3, n}, p\right)
\end{array}\right]
$$$$
:=\left[\begin{array}{l}
I_{r e f}-K\left(\Phi_{1}\left(t_{1, n}\right) x_{n}+\Psi_{1}\left(t_{1, n}\right)\right) \\
I_{r e f}-K\left(\Phi _ { 1 } ( t _ { 3 , n } - T ) \left(\Phi_{2}\left(T-t_{1, n}\right)\right.\right. \\
\left.\left(\Phi_{1}\left(t_{1, n}\right) x_{n}+\Psi_{1}\left(t_{1, n}\right)\right) \Psi_{2}\left(T-t_{1, n}\right)\right) \\
\left.+\Psi_{1}\left(t_{3, n}-T\right)\right)
\end{array}\right]
$$

$$
=\left[\begin{array}{l}
0 \\
0
\end{array}\right]
$$

3.2.1 Fixed points. Imposing the constraint $x_{n+1}=x_{n}=$ $x^{* *}$, the fixed points are given as follows:

$$
\begin{aligned}
x^{* *}= & \left(I-\Phi_{2}\left(2 T-t_{3}^{*}\right) \Phi_{1}\left(t_{3}^{*}-T\right) \Phi_{2}\left(T-t_{1}^{*}\right)\right. \\
& \left.\Phi_{1}\left(t_{1}^{*}\right)\right)^{-1}\left(\Phi_{2}\left(2 T-t_{3}^{*}\right) \Phi_{1}\left(t_{3}^{*}-T\right) \Phi_{2}\left(T-t_{1}^{*}\right)\right. \\
& \Psi_{1}\left(t_{1}^{*}\right)+\Phi_{2}\left(2 T-t_{3}^{*}\right) \Phi_{1}\left(t_{3}^{*}-T\right) \Psi_{2}\left(T-t_{1}^{*}\right) \\
& \left.+\Phi_{2}\left(2 T-t_{3}^{*}\right) \Psi_{1}\left(t_{3}^{*}-T\right)+\Psi_{2}\left(2 T-t_{3}^{*}\right)\right)
\end{aligned}
$$

where $\tau^{*}=\left[t_{1}^{*}, T, t_{3}^{*}\right]^{T}$ corresponding to the fixed point $x^{* *}$. The switching equations are:

$$
\begin{aligned}
& \sigma\left(x^{* *}, \tau^{*}, p\right):=\left[\begin{array}{c}
\sigma_{1}\left(x^{* *}, t_{1}^{*}, p\right) \\
\sigma_{2}\left(x^{* *}, t_{1}^{*}, t_{3}^{*}, p\right)
\end{array}\right] \\
& :=\left[\begin{array}{l}
I_{\text {ref }}-K\left(\Phi_{1}\left(t_{1}^{*}\right) x^{* *}+\Psi_{1}\left(t_{1}^{*}\right)\right) \\
I_{\text {ref }}-K\left(\Phi _ { 1 } ( t _ { 3 } ^ { * } - T ) \left(\Phi_{2}\left(T-t_{1}^{*}\right)(\right.\right. \\
\left.\Phi_{1}\left(t_{1}^{*}\right) x^{* *}+\Psi_{1}\left(t_{1}^{*}\right)\right)+\Psi_{2}\left(T-t_{1}^{*}\right) \\
)+\Psi_{1}\left(t_{3}^{*}-T\right)\right)
\end{array}\right] \\
& =\left[\begin{array}{l}
0 \\
0
\end{array}\right]
\end{aligned}
$$

The procedure for determining $\tau^{*}$ and $x^{* *}$ is the same as that used for the 1-periodic fixed points. We determine $\tau^{*}$ replacing Eq. (15) in Eq. (16). When $\tau^{*}$ is obtained we replace it in Eq. (15) to determine $x^{* *}$. 


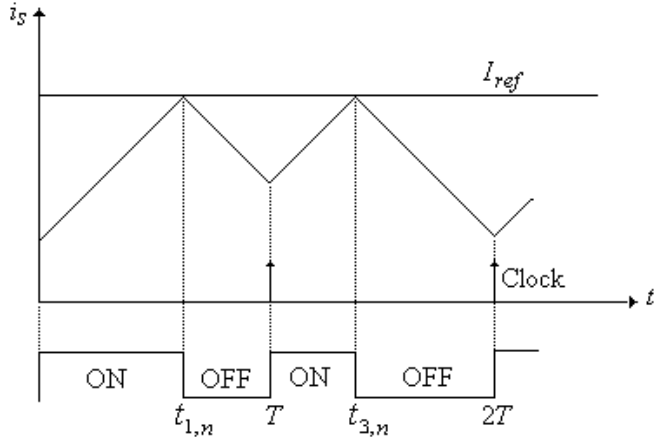

(a)

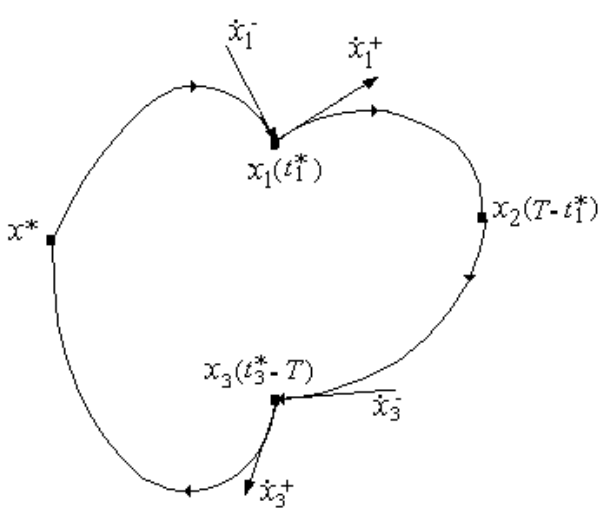

(c)

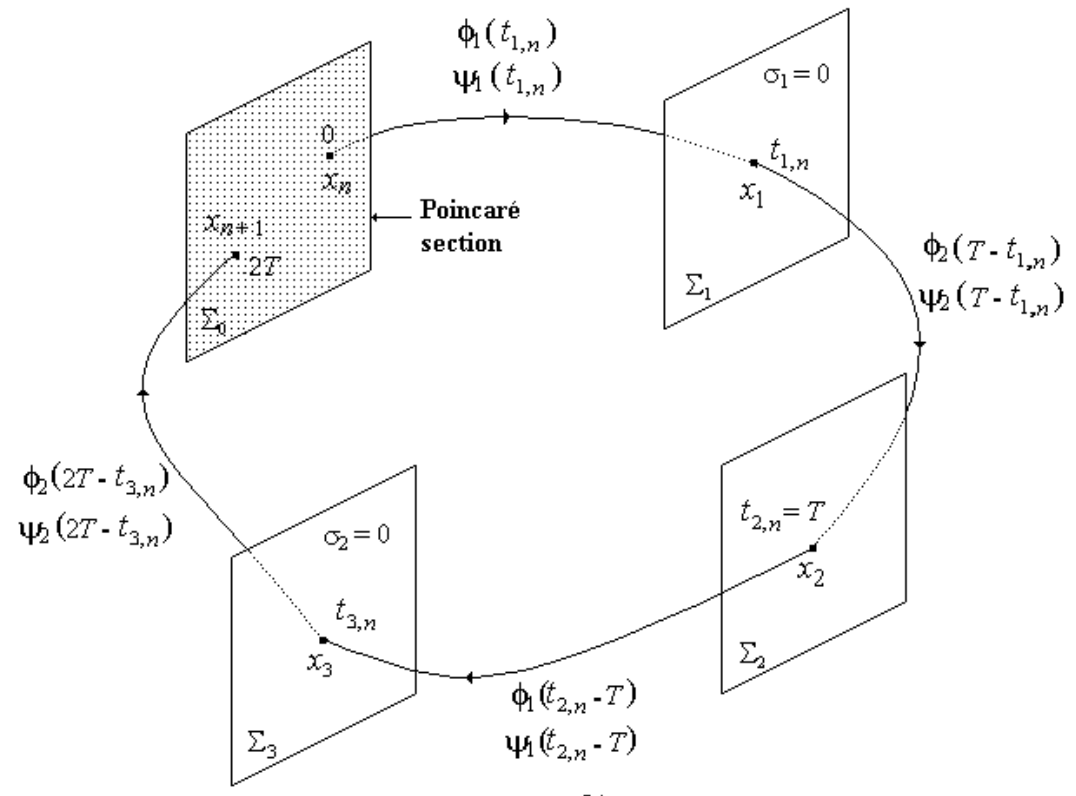

(b)

Fig. 6. (a) A stable 2-periodic behavior.(b) Schematic mapping of 2-periodic orbit's nonlinear discrete map. (c) Typical 2-periodic solution.

3.2.2 Jacobian matrix. The Jacobian matrix $D P$ of Eq. (13) and Eq. (14) evaluated at $x^{* *}$ is given as follows:

$$
D P=\frac{\partial P}{\partial x_{n}}-\left.\frac{\partial P}{\partial \tau_{n}}\left(\frac{\partial \sigma}{\partial \tau_{n}}\right)^{-1} \frac{\partial \sigma}{\partial x_{n}}\right|_{x^{* *}, \tau^{*}}
$$

Evaluating each partial derivative and replacing it in Eq. (17), the Jacobian matrix $D P$ evaluated at $x^{* *}$ becomes:

$$
\begin{aligned}
D P= & \Phi_{2}\left(2 T-t_{3}^{*}\right)\left(I-\left(\frac{\left(\dot{x}_{1}^{-}-\dot{x}_{1}^{+}\right)}{K \dot{x}_{1}^{-}}-\frac{\left(\dot{x}_{1}^{-}-\dot{x}_{1}^{+}\right)\left(\dot{x}_{3}^{-}-\dot{x}_{3}^{+}\right)}{K \dot{x}_{1}^{-} \dot{x}_{3}^{-}}\right) K\right. \\
& \left.-\frac{\dot{x}_{3}^{-}-\dot{x}_{3}^{+}}{\dot{x}_{3}^{-}}\right) \Phi_{1}\left(t_{3}^{*}-T\right) \Phi_{2}\left(T-t_{1}^{*}\right) \Phi_{1}\left(t_{1}^{*}\right)
\end{aligned}
$$

where $\dot{x}_{1}^{-}, \dot{x}_{1}^{+}$and $\dot{x}_{3}^{-}, \dot{x}_{3}^{+}$are the time derivatives of $x$ at switching times $t_{1}^{*}$ and $t_{3}^{*}$ respectively (Fig.6-c) which can be written, as follows:

$$
\begin{aligned}
\dot{x}_{1}^{-}= & A_{1}\left(\Phi_{1}\left(t_{1}^{*}\right) x^{* *}+\Psi_{1}\left(t_{1}^{*}\right)\right)+B_{1} \\
\dot{x}_{1}^{+}= & A_{2}\left(\Phi_{1}\left(t_{1}^{*}\right) x^{* *}+\Psi_{1}\left(t_{1}^{*}\right)\right)+B_{2} \\
\dot{x}_{3}^{-}= & A_{1}\left(\Phi _ { 1 } ( t _ { 3 } ^ { * } - T ) \left(\Phi_{2}\left(T-t_{1}^{*}\right)\left(\Phi_{1}\left(t_{1}^{*}\right) x^{* *}+\Psi_{1}\left(t_{1}^{*}\right)\right)\right.\right. \\
& \left.\left.+\Psi_{2}\left(T-t_{1}^{*}\right)\right)+\Psi_{1}\left(t_{3}^{*}-T\right)\right)+B_{1} \\
\dot{x}_{3}^{+}= & A_{2}\left(\Phi _ { 1 } ( t _ { 3 } ^ { * } - T ) \left(\Phi_{2}\left(T-t_{1}^{*}\right)\left(\Phi_{1}\left(t_{1}^{*}\right) x^{* *}+\Psi_{1}\left(t_{1}^{*}\right)\right)\right.\right. \\
& \left.\left.+\Psi_{2}\left(T-t_{1}^{*}\right)\right)+\Psi_{1}\left(t_{3}^{*}-T\right)\right)+B_{2}
\end{aligned}
$$

3.2.3 Stability Study of 2-Periodic Orbit. The stability of 2periodic orbit is carried out by using Eq. (18). Figure 7 shows the evolution of the eigenvalues of Jacobian matrix evaluated at 2-periodic fixed points when $I_{r e f}$ is varied in the range $(4,6) \mathrm{A}$ . We can see that all of the eigenvalues are inside the unit circle and this shows that the 2-periodic orbit is stable. However, by means of the PSIM simulation, it is obtained that the system exhibits chaos when $I_{r e f}$ is increased (Fig. 8). In order to explain this phenomenon, the evolution of the duty cycles cor- responding to the fixed point of the second order Poincare map with $I_{r e f}$ is shown in Fig. 9. This figure shows that for a certain critical value of $I_{r e f}$, the duty cycles are at outside the range $(0,1)$ which is the allowed interval for this variable. This means that, at the critical value of bifurcation parameter, a saturation of the duty cycle will take place in the real system. Evidently, this saturation is not taken into account in the previous map (Eq.(5)) which was constructed supposing that the number of configurations is the same during one switching cycle. However, due to the saturation effect one of the configurations may disappear. As it will be seen, this is the cause of an anomalous (border collision) bifurcation (BCB) [12 13]. This kind of bifurcations cannot be detected using the previous smooth map. Therefore, a more realistic map is needed to plot the true bifurcation diagram of the system when the number of configurations can change. This model will be presented in the following paragraph.

\section{BIFURCATION ANALYSIS}

Knowing that DC-DC converters are very used in many industrial, bifurcation analysis has became a useful tool for power converter designers [14, 15].

The map (Eq. (5)) is only valid when $i_{s}$ reaches $I_{r e f}$ before the arrival of the pulse clock. But if the pulse clock arrives just or $i_{s}$ is reaches $I_{r e f}$, the map can be described by the following equation:

$$
\begin{aligned}
& x_{n+1}:=\Phi_{1}(T) x_{n}+\Psi_{1}(T) \\
& \sigma\left(x_{n}, \tau_{n}, p\right):=I_{r e f}-K\left(\Phi_{1}(T) x_{n}+\Psi_{1}(T)\right)=0
\end{aligned}
$$

The borderline (BL) between the two regions (Eq. (5) and Eq. (19) corresponds to the case when $i_{s}$ reaches $I_{\text {ref }}$ at just the arrival of the pulse clock. Taking into account the above operating conditions, the map has a global expression in the following 


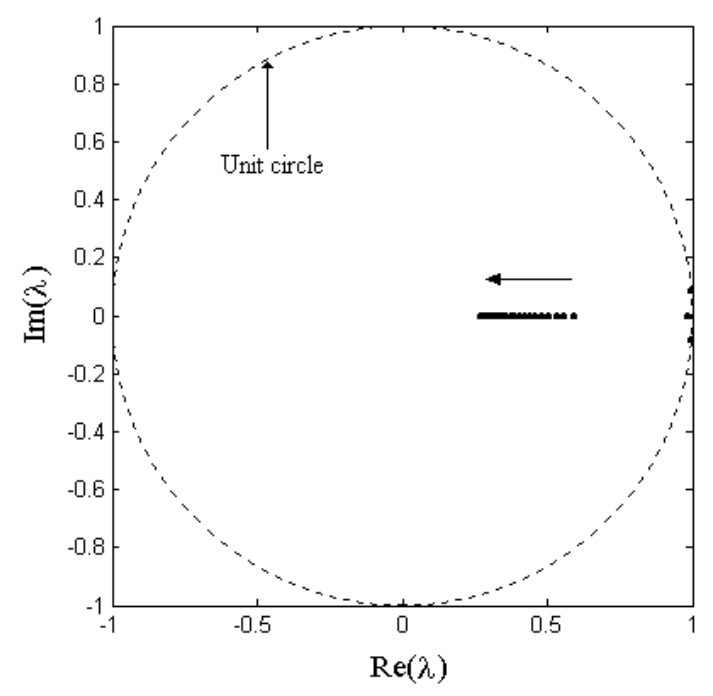

Fig. 7. The evolution of eigenvalues of the Jacobian matrix evaluated at the 2-periodic fixed points when $I_{r e f}$ is varied in the range $(4,6) \mathbf{A}$.

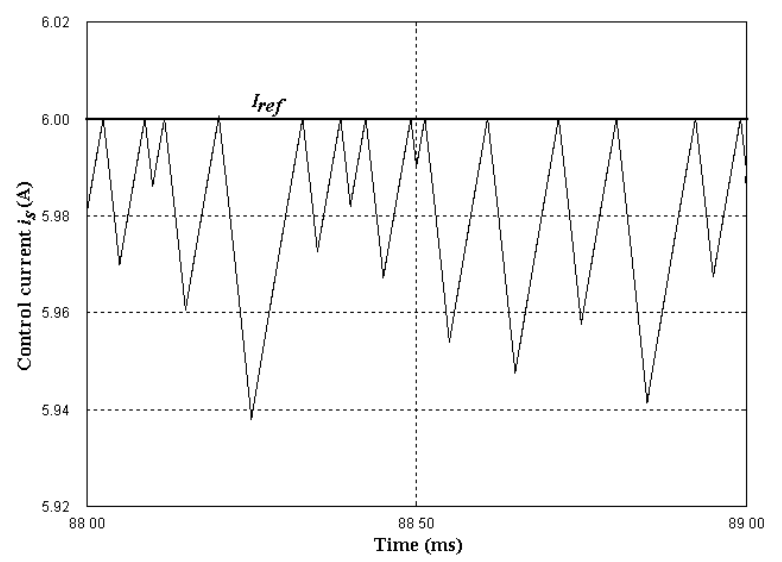

Fig. 8. Time domain waveform of the control signal $i_{s}$ at $I_{r e f}=6$ A (PSIM simulation).

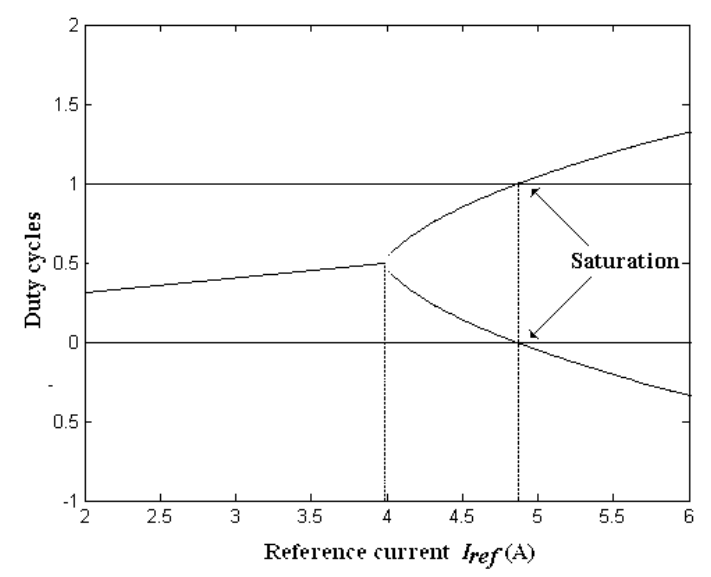

Fig. 9. The evolution of duty cycles when $I_{r e f}$ is varied in the range $(2,6) \mathbf{A}$.

form:

$$
x_{n+1}= \begin{cases}f\left(x_{n}, p\right) & \text { if } i_{s}<I_{\text {border }} \\ g\left(x_{n}, p\right) & \text { if } i_{s}>I_{\text {border }}\end{cases}
$$

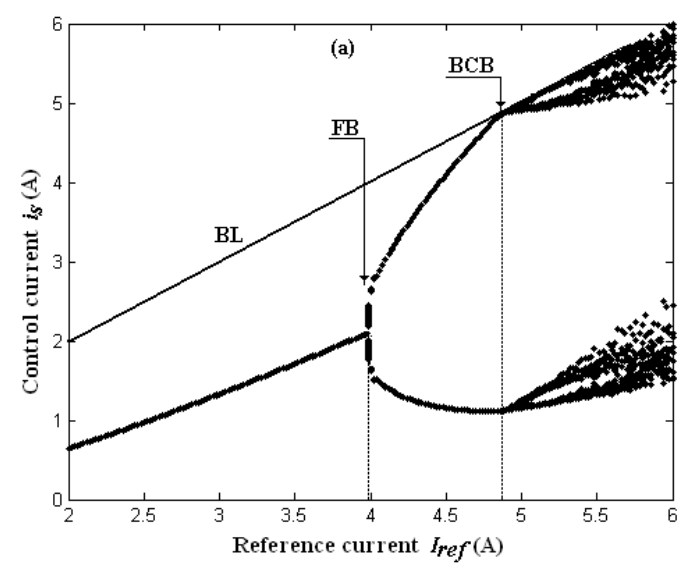

Fig. 10. Bifurcation diagram of control current $i_{s}$ taking $I_{r e f}$ as a bifurcation parameter.

where $f(),. g($.$) are two vector functions defined by Eq. (5) and$ Eq. (19) respectively.

\subsection{Determination of the borderline $I_{b o r d e r}$}

Generally the switching period is so small that $\Phi_{1}(T)$ and $\Psi_{1}(T)$ of Eq. (19), can be approximated by the first few terms in the expansion of matrix exponential.

$$
\begin{aligned}
& \Phi_{1}(T) \approx I+A_{1} T \\
& \Psi_{1}(T) \approx B_{1} T
\end{aligned}
$$

Replacing Eq. (21) in Eq. (19) we can obtain the following approximated switching equation just at the arrival of the clock:

$$
\begin{aligned}
& I_{r e f}-\left(\left(1-\frac{r_{L_{2}}}{L_{2}} T\right) i_{L_{2}}+\left(1-\frac{r_{L_{1}}}{L_{2}} T\right) i_{L-1}+\frac{v_{C_{1}}}{L_{2}} T\right. \\
& \left.-\frac{v_{C_{2}}}{L_{1}} T+\frac{V_{i n}}{L_{1}} T\right)=0
\end{aligned}
$$

Being $r_{L_{1}}=r_{L_{2}}, L_{1}=L_{2}$ and $v_{C_{1}}=V_{i n}+v_{C_{2}}$ (at steady state), Eq. (22) is simplified and the borderline is given as follows:

$$
I_{\text {border }}=\frac{I_{r e f}-\frac{2 V_{i n}}{L_{1}} T}{1-\frac{r_{L_{1}} T}{L_{1}}}
$$

Using the complete model (Eq. (20)), the bifurcation diagrams of $i_{s}$ is shown in Fig. 10. We can see that for low values of $I_{r e f}$, the system dynamic behavior is a stable 1-periodic orbit. As $I_{r e f}$ is increased, this orbit becomes unstable due to a FB. Consequently, a stable 2-periodic orbit takes place. This bifurcation occurs at a critical value of $I_{r e f, c r i} \simeq 4 \mathrm{~A}$. By further increasing $I_{r e f}$, the 2-periodic orbit collides with the BL. This results in a BCB at a value of $I_{r e f} \simeq 4.8 \mathrm{~A}$. The different regimes of the converter are illustred in Fig.11-a, Fig.11-b, and Fig.11-c. In these figures, the waveforms and the phase portrait are presented.

\section{CONCLUSIONS}

In this work, bifurcation analysis of current mode control Ćuk DC-DC converter operating in conduction continuous mode is carried out. This analysis has been performed by using the nonlinear discrete maps of as much for 1-periodic orbit as for 2periodic orbit. When the reference current is varied, it has shown that the 1-periodic orbit loses via flip bifurcation. Consequently, a stable 2-periodic orbit takes place. When the reference current exceeds critical value, 2-periodic orbit collides with borderline and bifurcates to chaos via border collision bifurcation. A closed form expression of borderline has been calculated. 

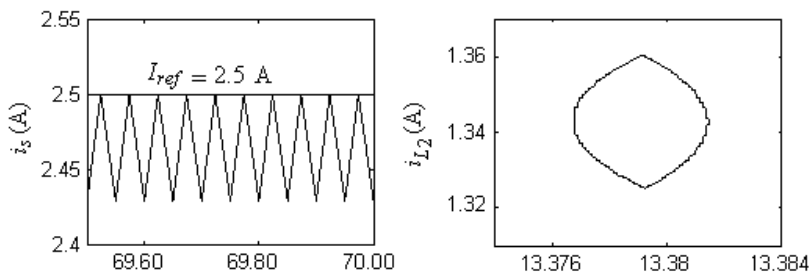

(a)
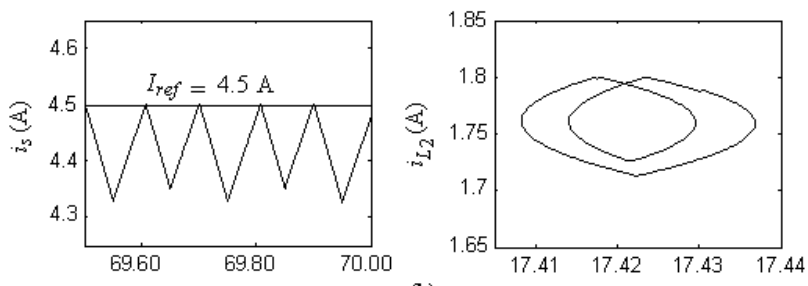

(b)
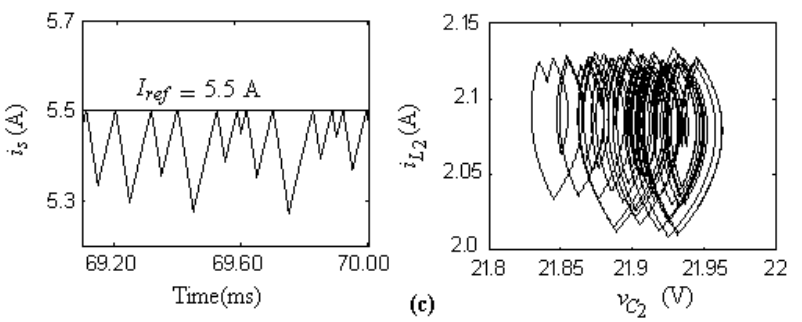

Fig. 11. (a) The 1-periodic regime $\left(I_{r e f}=2.5 \mathrm{~A}\right)$. (b) The 2-periodic regime $\left(I_{r e f}=4.5 \mathrm{~A}\right)$. (c) The chaotic regime $\left(I_{\text {ref }}=5.5 \mathbf{A}\right)$.

\section{REFERENCES}

[1] J. H. Deane and D. C. Hamill, "Chaotic behaviour in current mode controlled DC-DC converter," Electronics Letters, vol. 27, pp. 1172-1173, 1991.

[2] M. di Bernardo, F. Grofalo, L. Glielmo, and F. Vasca, "Quasiperiodic behaviors in DC-DC converters," in PESC'96: Power Electronics Specialist Conferences, pp. 1376-1381, 1996.

[3] K. W. E. Cheng, M. Liu, J. Wu, and N. C. Cheung, "Study of bifurcation and chaos in the current- mode controlled buck-boost DC-DC converter,' in IECON'01: Industrial Electronics Society Conference., pp. 838-343, 2001.

[4] A. E. Aroudi, M. B. Debbat, and L. Martínez-Salamero, "Poincaré maps modeling and local orbital stability analysis of discontinuous piecewise affine periodically driven systems," Nonlinear Dynamics, vol. 50, pp. 431-445, 2007.
[5] C. K. Tse, S. C. Fung, and M. W. Kwan, "Experimental confirmation of chaos in a current-programmed Cuk converter," IEEE Trans. on Circuits and Systems-I, vol. 43, pp. 605-608, 1996.

[6] C. Y. William and C. K. Tse, "Study of bifurcations in current-programmed DC-DC converters: From quasiperiodicity to period-doubling," IEEE Trans. on Circuits and Systems-I, vol. 44, pp. 1129-1142, 1997.

[7] C. K. Tse, Y. M. Lai, and H. H. C. Iu, "Hopf bifurcation and chaos in a free-running autonomous uk converter," IEEE Trans. on Circuits and Systems-I, vol. 47, pp. 448-457, 2000.

[8] C. M. Ivan, D. Lascu, and V. Popescu, "Hopf bifurcation in a discontinuous capacitor voltage mode uk DCDC converter," in The 6th WSEAS International Conference on Instrumentation, Measurement, Circuits and Systems, (Hangzhou), pp. 431-445, 2007.

[9] G. Verghese, M. Elbuluk, , and J. Kassakian, "A general approach to sample-data modeling for power electronic circuits," IEEE Transactions on Power Electronics, vol. 1, no. 2, pp. 76-89, 1986.

[10] M. B. Debbat, A. E. Aroudi, R. Giral, and L. MartínezSalamero, "Stability analysis and bifurcations of sepic DC-DC converter using a discrete-time model," in ICIT'02: International Conference on Industrial Technology, (Bangkok), pp. 1055-1060, 2002.

[11] W. Ma, M. Wang, and C. Li, "Control of bifurcation in the one-cycle controlled cuk converter," Nonlinear Dynamics, vol. 67, pp. 2573-2583, March 2012.

[12] M. di Bernardo, F. Garofalo, L. Glielmo, and F. Vasca, "Switchings, bifurcations and chaos in dc-dc converters," IEEE Trans. on Circuits and Systems-I, vol. 45, no. 2, pp. 133-141, 1998.

[13] Z. T. Zhusubaliyev, E. Soukhoterin, and E. Mosekilde, "Quasiperiodicity and border-collision bifurcations in a dcdc converter with pulsewidth modulation," IEEE Trans. on Circuits and Systems-I, vol. 50, pp. 1047-1057, August 2003.

[14] A. E. Aroudi, E. Rodriguez, R. Leyva, and E. Alarcon, "A design-oriented combined approach for bifurcation prediction in switched-mode power converters," IEEE Trans. on Circuits and Systems-II, vol. 57, pp. 218-222, 2010.

[15] M. Sattler, "Analysis of bifurcation behavior in power converters and the impact on the design process," in ESTS'11: IEEE Electric Ship Technologies Symposium, (Alexandria, VA), pp. 203-206, 2011. 